\title{
Surgical trainee impact on bariatric surgery safety
}

\author{
Iliya Goldberg ${ }^{1}$ (I) Jie Yang ${ }^{2}$ - Jihye Park ${ }^{3}$. Aurora D. Pryor ${ }^{1} \cdot$ Salvatore Docimo Jr. ${ }^{1}$. Andrew T. Bates ${ }^{1}$. \\ Mark A. Talamini ${ }^{1} \cdot$ Konstantinos Spaniolas $^{1}$
}

Received: 4 May 2018 / Accepted: 7 November 2018 / Published online: 13 November 2018

(c) Springer Science+Business Media, LLC, part of Springer Nature 2018

\begin{abstract}
Background Roux-en-Y-gastric bypass (RYGB) and sleeve gastrectomy (SG) are commonly performed bariatric procedures that are associated with a significant learning curve. The effect of surgeon experience on perioperative outcomes and safety is established, but the effect of trainee participation remains unclear. The purpose of this study was to assess the impact of trainees on early perioperative safety of bariatric surgery.

Methods Metabolic and Bariatric Surgery Accreditation and Quality Improvement Program database for 2015 was used to identify non-revisional laparoscopic and robotic RYGB and SG procedures. Comparisons were made based on assistant level. Multivariable logistic and linear regression methodology was used to compare clinical outcomes.

Results There were 35,354 laparoscopic RYGB, 2896 robotic RYGB, 79,717 laparoscopic SG, and 5449 robotic SG procedures examined. $21,257(17 \%)$ and 11,322 (9\%) of all procedures were performed with a resident or fellow, respectively. Fellow presence was independently associated with the development of complications for all procedure types except robotic SG when compared to non-trainee [odds ratio (OR) 1.31, 2.20, 1.28 for laparoscopic RYGB, robotic RYGB, and laparoscopic SG, respectively]. The most common events were urinary tract and superficial surgical site infections. This negative impact of fellow on overall complications was eliminated after accounting for operative duration. In laparoscopic SG, resident participation was associated with higher leak rate (OR 1.61), readmission (OR 1.18), re-intervention (OR 1.4), and complication rate (OR 1.32) compared to non-trainee, even after accounting for procedural duration. In robotic SG, there was no impact of trainee on outcomes.

Conclusions Although fellow training is associated with higher overall complication rate, there is no such impact on major safety benchmarks, including leak rate and re-operation. In contrast, the impact of resident trainees on SG outcomes is substantial. Operative involvement of trainees in bariatric surgery leads to different outcomes based on trainee level and should be judiciously considered during the programmatic design of bariatric educational curricula.
\end{abstract}

Keywords Bariatric $\cdot$ Sleeve gastrectomy $\cdot$ Roux-en-Y-gastric bypass $\cdot$ Fellowship training $\cdot$ Robotic $\cdot$ Resident training

Electronic supplementary material The online version of this article (https://doi.org/10.1007/s00464-018-6587-0) contains supplementary material, which is available to authorized users.

Konstantinos Spaniolas

konstantinos.spaniolas@stonybrookmedicine.edu

1 Division of Bariatric, Foregut and Advanced Gastrointestinal Surgery, Department of Surgery, Stony Brook University Medical Center, 100 Nichols Road, HSC T19, Stony Brook, NY 11794, USA

2 Department of Family, Population and Preventive Medicine, Stony Brook University Medical Center, Stony Brook, NY 11794, USA

3 Department of Applied Mathematics and Statistics, Stony Brook University, Stony Brook, NY 11794, USA
Since its original inception in the 1950s, the field of bariatric surgery has become increasingly more popular in large part due to its superior impacts on weight loss, hyperglycemia control, hyperlipidemia, hypertension, cardiovascular risk, and mortality outcomes as compared to medical therapy alone $[1,2]$. The most commonly performed bariatric procedures include laparoscopic sleeve gastrectomy (SG) and laparoscopic Roux-en-Y-gastric bypass (RYGB) [3]. Bariatric procedures are technically complex involving certain key steps and intricacies to prevent debilitating morbidity and mortality $[4,5]$. Fellowship training has become a significant component of bariatric practice in the US [6] and has been suggested to lead to improved perioperative outcomes and decreased procedural duration for RYGB [4, 7]. 
Surgical training has been under great scrutiny as healthcare moves toward quantifying patient safety, value, and surgical outcomes [8]. There have been numerous studies involving resident and fellow participation among general surgery and several subspecialties with variable results; some showing worse outcomes $[9,10]$ and others showing no difference $[11,12]$. Most of the negative effect seen with resident and fellow participation was primarily based on prolonged operative duration [10, 13]. A few studies, however, showed increased overall morbidity rate in various general surgery procedures $[9,14]$. Interestingly, several of the studies showed that trainee participation did not negatively impact morbidity or mortality and deemed that resident participation is overall safe for surgical patients [14-16].

Trainee participation in laparoscopic SG has been associated with increased readmissions, urinary tract infections (UTIs), pulmonary emboli, surgical site infections (SSIs), and prolonged operative times [17]. These outcomes were comparable between the resident and fellow groups [17]. In a study by Aminian et al., while fellow participation in laparoscopic SG was associated with prolonged operative time when compared to a non-trainee, there was no increased rate of postoperative complications. On the other hand, fellow involvement in laparoscopic RYGB was associated with a higher rate of overall complications, serious complications as well as 30-day re-operations [18].

Robotic surgery has become an attractive endeavor for many surgeons as it often provides a shorter learning curve, three-dimensional optics, improved ergonomics, and wristed instrumentation [19]. Few studies comparing laparoscopic and robotic SG have demonstrated that complications are comparable between the two approaches [20]. Using the robotic platform for resident teaching during SG has demonstrated outcomes similar to those for laparoscopic SG [21]. Despite the novelty of this technology, Vilallonga et al. suggested that the learning curve for robotic SG is only 20 cases [22]. Similarly, robotic RYGB has been shown to have a shorter learning curve when compared to the laparoscopic approach [23].

The aim of this study was to assess the impact of trainee operative involvement on early perioperative safety of bariatric surgery. Secondarily, we aimed to assess the differential impact between resident and fellow participation in robotic bariatric surgery and how their participation influences outcomes and patient safety.

\section{Methods}

After Institutional Review Board approval was obtained, the Metabolic and Bariatric Surgery Accreditation and Quality Improvement Program 2015 public use file was used to identify non-revisional laparoscopic and robotic RYGB and SG procedures. Subject consent was waived given the statewide nature of the study and the use of deidentified data. Comparisons were made based on the type of assistant level listed in the database: resident, fellow, or non-trainee (including an attending surgeon, nurse first assistant, or physician extenders). Baseline pre-operative data included patient demographics, body mass index (BMI), comorbidities, smoking status, and American Society of Anesthesiology (ASA) score. Complications resulting in death or unfavorable occurrences were examined as previously described [24]. Clinical outcomes included anastomotic/staple line leak, 30-day readmission, 30-day re-operation, 30-day intervention, any complication, and procedural duration.

For the statistical analysis, $\chi^{2}$ tests with exact $p$ values based on Monte Carlo simulation were used to examine unadjusted marginal association between each categorical variable and clinical outcome such as anastomotic/staple line leak, 30-day readmission, 30-day re-operation, 30-day intervention, and any complication. Wilcoxon rank-sum tests were used to compare unadjusted marginal differences for any continuous variable between groups. Non-parametric tests, such as Wilcoxon rank-sum tests or Kruskal-Wallis tests were used to compare unadjusted marginal differences in continuous outcomes (operation length) by each categorical variable, and Spearman's rank correlation coefficients were used to check the relationship between two continuous outcomes. Any factors related to each outcome that were significant ( $p$ value $<0.1$ ) based on univariate analysis were further adjusted for in multivariable regression models. Multivariable logistic regression models were used to compare the risk of anastomotic/staple line leak, 30-day readmission, 30-day re-operation, 30-day intervention, and any complication, while multivariable linear regression model was used to compare operation length where log-transformation was used to make the model assumption met (see Appendix A1). In each logistic regression analysis, an odds ratio $(\mathrm{OR})>1$ indicates that one category has more risk of having an outcome than the reference category, and $\mathrm{OR}<1$ indicates that one category has less risk of having an outcome than the reference category. Statistical analysis was performed using SAS 9.4 and significance level was set at 0.05 (SAS Institute, Inc., Cary, NC).

\section{Results}

\section{General}

There were 35,354 laparoscopic RYGB, 2896 robotic RYGB, 79,717 laparoscopic SG, and 5449 robotic SG procedures examined. Residents or fellows were participating in 21,257 $(17 \%)$ and $11,322(9 \%)$ of all the procedures, respectively (Table 1). 
Table 1 Descriptive table of baseline demographics and comorbidities for all bariatric patients by assistant participation

\begin{tabular}{|c|c|c|c|c|c|c|c|}
\hline Variables & Missing & Levels & Total & $\begin{array}{l}\text { Resident } \\
(\mathrm{N}=21,257)\end{array}$ & $\begin{array}{l}\text { Minimally inva- } \\
\text { sive surgery fellow } \\
(\mathrm{N}=11,322)\end{array}$ & $\begin{array}{l}\text { Non-trainee } \\
(\mathrm{N}=90,810)\end{array}$ & p-values* \\
\hline \multicolumn{8}{|l|}{$\begin{array}{l}\text { Patients' character- } \\
\text { istics }\end{array}$} \\
\hline Age & 0 & $\begin{array}{l}21,257 \text { versus } \\
11,322 \text { versus } \\
90,810\end{array}$ & $44.00 \pm 17.00$ & $44.00 \pm 18.00$ & $44.00 \pm 18.00$ & $44.00 \pm 17.00$ & $<0.0001$ \\
\hline \multirow[t]{2}{*}{ Sex } & \multirow[t]{2}{*}{0} & Female & $97,245(78.81 \%)$ & $16,975(79.86 \%)$ & $8821(77.91 \%)$ & $71,449(78.68 \%)$ & \multirow[t]{2}{*}{$<0.0001$} \\
\hline & & Male & $26,144(21.19 \%)$ & $4282(20.14 \%)$ & $2501(22.09 \%)$ & $19,361(21.32 \%)$ & \\
\hline \multirow[t]{3}{*}{ Race } & \multirow[t]{3}{*}{0} & Black & $21,123(17.12 \%)$ & $4524(21.28 \%)$ & $2398(21.18 \%)$ & $14,201(15.64 \%)$ & \multirow[t]{3}{*}{$<0.0001$} \\
\hline & & Other & $10,163(8.24 \%)$ & $2835(13.34 \%)$ & $1262(11.15 \%)$ & $6066(6.68 \%)$ & \\
\hline & & White & $92,103(74.64 \%)$ & $13,898(65.38 \%)$ & $7662(67.67 \%)$ & $70,543(77.68 \%)$ & \\
\hline $\begin{array}{l}\text { Pre-Op BMI clos- } \\
\text { est to bariatric } \\
\text { surgery }\end{array}$ & 0 & $\begin{array}{l}21,257 \text { versus } \\
11,322 \text { versus } \\
90,810\end{array}$ & $44.00 \pm 9.72$ & $44.21 \pm 9.53$ & $44.35 \pm 9.74$ & $43.90 \pm 9.74$ & $<0.0001$ \\
\hline \multicolumn{8}{|l|}{ Comorbidities } \\
\hline \multirow[t]{4}{*}{ ASA class } & \multirow[t]{4}{*}{317} & No disturb & $484(0.39 \%)$ & $168(0.79 \%)$ & $31(0.27 \%)$ & $285(0.31 \%)$ & \multirow[t]{4}{*}{$<0.0001$} \\
\hline & & Mild disturb & $28,457(23.12 \%)$ & $5579(26.27 \%)$ & $2710(23.97 \%)$ & $20,168(22.28 \%)$ & \\
\hline & & Severe disturb & $89,435(72.67 \%)$ & $14,711(69.27 \%)$ & $8263(73.07 \%)$ & $66,461(73.42 \%)$ & \\
\hline & & $\begin{array}{l}\text { Life threat, mori- } \\
\text { bund }\end{array}$ & $4696(3.82 \%)$ & $779(3.67 \%)$ & $304(2.69 \%)$ & $3613(3.99 \%)$ & \\
\hline \multirow{2}{*}{$\begin{array}{l}\text { Exceptionally } \\
\text { long operation }\end{array}$} & \multirow[t]{2}{*}{0} & No & $92,240(74.76 \%)$ & $13,141(61.82 \%)$ & $6492(57.34 \%)$ & $72,607(79.95 \%)$ & \multirow[t]{2}{*}{$<0.0001$} \\
\hline & & Yes & $31,149(25.24 \%)$ & $8116(38.18 \%)$ & $4830(42.66 \%)$ & $18,203(20.05 \%)$ & \\
\hline \multirow{2}{*}{$\begin{array}{l}\text { Pre-Op steroid/ } \\
\text { immunosup- } \\
\text { pressant use } \\
\text { for chronic } \\
\text { condition }\end{array}$} & \multirow[t]{2}{*}{0} & No & $121,465(98.44 \%)$ & $20,880(98.23 \%)$ & $11,126(98.27 \%)$ & $89,459(98.51 \%)$ & \multirow[t]{2}{*}{0.0031} \\
\hline & & Yes & $1924(1.56 \%)$ & $377(1.77 \%)$ & $196(1.73 \%)$ & $1351(1.49 \%)$ & \\
\hline \multirow{2}{*}{$\begin{array}{l}\text { Pre-Op history of } \\
\text { COPD }\end{array}$} & \multirow[t]{2}{*}{0} & No & $121,148(98.18 \%)$ & $20,893(98.29 \%)$ & $11,086(97.92 \%)$ & $89,169(98.19 \%)$ & \multirow[t]{2}{*}{0.0525} \\
\hline & & Yes & $2241(1.82 \%)$ & $364(1.71 \%)$ & $236(2.08 \%)$ & $1641(1.81 \%)$ & \\
\hline \multirow{2}{*}{$\begin{array}{l}\text { Pre-Op diabetes } \\
\text { mellitus }\end{array}$} & \multirow[t]{2}{*}{0} & No & $90,039(72.97 \%)$ & $15,507(72.95 \%)$ & $8020(70.84 \%)$ & $66,512(73.24 \%)$ & \multirow[t]{2}{*}{$<0.0001$} \\
\hline & & Yes & $33,350(27.03 \%)$ & $5750(27.05 \%)$ & $3302(29.16 \%)$ & $24,298(26.76 \%)$ & \\
\hline \multirow{2}{*}{$\begin{array}{l}\text { Pre-Op requiring } \\
\text { or on dialysis }\end{array}$} & \multirow[t]{2}{*}{0} & No & $123,069(99.74 \%)$ & $21,175(99.61 \%)$ & $11,268(99.52 \%)$ & $90,626(99.80 \%)$ & \multirow[t]{2}{*}{$<0.0001$} \\
\hline & & Yes & $320(0.26 \%)$ & $82(0.39 \%)$ & $54(0.48 \%)$ & $184(0.20 \%)$ & \\
\hline Pre-Op functional & 0 & Independent & $122,141(98.99 \%)$ & 21,063 (99.09\%) & $11,190(98.83 \%)$ & $89,888(98.98 \%)$ & 0.0011 \\
\hline health status & & Partially dependent & $846(0.69 \%)$ & $153(0.72 \%)$ & $81(0.72 \%)$ & $612(0.67 \%)$ & \\
\hline & & Totally dependent & $402(0.33 \%)$ & $41(0.19 \%)$ & $51(0.45 \%)$ & $310(0.34 \%)$ & \\
\hline Pre-Op GERD & 0 & No & $84,955(68.85 \%)$ & $14,849(69.85 \%)$ & $7655(67.61 \%)$ & $62,451(68.77 \%)$ & 0.0001 \\
\hline $\begin{array}{l}\text { requiring medi- } \\
\text { cation }\end{array}$ & & Yes & $38,434(31.15 \%)$ & $6408(30.15 \%)$ & $3667(32.39 \%)$ & $28,359(31.23 \%)$ & \\
\hline Pre-Op hyperten- & 0 & No & $61,790(50.08 \%)$ & $10,977(51.64 \%)$ & $5526(48.81 \%)$ & $45,287(49.87 \%)$ & $<0.0001$ \\
\hline $\begin{array}{l}\text { sive requiring } \\
\text { medication }\end{array}$ & & Yes & $61,599(49.92 \%)$ & $10,280(48.36 \%)$ & $5796(51.19 \%)$ & $45,523(50.13 \%)$ & \\
\hline Pre-Op vein & 0 & No & $121,422(98.41 \%)$ & $20,885(98.25 \%)$ & $11,105(98.08 \%)$ & $89,432(98.48 \%)$ & 0.0008 \\
\hline $\begin{array}{l}\text { thrombosis } \\
\text { requiring } \\
\text { therapy }\end{array}$ & & Yes & $1967(1.59 \%)$ & $372(1.75 \%)$ & $217(1.92 \%)$ & $1378(1.52 \%)$ & \\
\hline History of PE & 0 & No & $122,036(98.90 \%)$ & $20,997(98.78 \%)$ & $11,161(98.58 \%)$ & $89,878(98.97 \%)$ & 0.0001 \\
\hline & & Yes & $1353(1.10 \%)$ & $260(1.22 \%)$ & $161(1.42 \%)$ & $932(1.03 \%)$ & \\
\hline Pre-Op hyperlipi- & 0 & No & $92,128(74.66 \%)$ & $15,938(74.98 \%)$ & $8262(72.97 \%)$ & $67,928(74.80 \%)$ & $<0.0001$ \\
\hline demia & & Yes & $31,261(25.34 \%)$ & $5319(25.02 \%)$ & $3060(27.03 \%)$ & $22,882(25.20 \%)$ & \\
\hline History of MI & 0 & No & $121,752(98.67 \%)$ & $20,949(98.55 \%)$ & $11,149(98.47 \%)$ & $89,654(98.73 \%)$ & 0.0189 \\
\hline & & Yes & $1637(1.33 \%)$ & $308(1.45 \%)$ & $173(1.53 \%)$ & $1156(1.27 \%)$ & \\
\hline
\end{tabular}


Table 1 (continued)

\begin{tabular}{|c|c|c|c|c|c|c|c|}
\hline Variables & Missing & Levels & Total & $\begin{array}{l}\text { Resident } \\
(\mathrm{N}=21,257)\end{array}$ & $\begin{array}{l}\text { Minimally inva- } \\
\text { sive surgery fellow } \\
(\mathrm{N}=11,322)\end{array}$ & $\begin{array}{l}\text { Non-trainee } \\
(\mathrm{N}=90,810)\end{array}$ & p-values* \\
\hline $\begin{array}{l}\text { Patient's ambula- } \\
\text { tion limited } \\
\text { most or all of } \\
\text { the time Pre-Op }\end{array}$ & 0 & $\begin{array}{l}\text { No } \\
\text { Yes }\end{array}$ & $\begin{array}{l}120,925(98.00 \%) \\
2464(2.00 \%)\end{array}$ & $\begin{array}{l}20,937(98.49 \%) \\
320(1.51 \%)\end{array}$ & $\begin{array}{l}11,079(97.85 \%) \\
243(2.15 \%)\end{array}$ & $\begin{array}{l}88,909(97.91 \%) \\
1901(2.09 \%)\end{array}$ & $<0.0001$ \\
\hline $\begin{array}{l}\text { Pre-Op oxygen } \\
\text { dependent }\end{array}$ & 0 & $\begin{array}{l}\text { No } \\
\text { Yes }\end{array}$ & $\begin{array}{l}122,514(99.29 \%) \\
875(0.71 \%)\end{array}$ & $\begin{array}{l}21,097(99.25 \%) \\
160(0.75 \%)\end{array}$ & $\begin{array}{l}11,240(99.28 \%) \\
82(0.72 \%)\end{array}$ & $\begin{array}{l}90,177(99.30 \%) \\
633(0.70 \%)\end{array}$ & 0.6711 \\
\hline $\begin{array}{l}\text { Previous cardiac } \\
\text { surgery }\end{array}$ & 0 & $\begin{array}{l}\text { No } \\
\text { Yes }\end{array}$ & $\begin{array}{l}121,989(98.87 \%) \\
1400(1.13 \%)\end{array}$ & $\begin{array}{l}21,005(98.81 \%) \\
252(1.19 \%)\end{array}$ & $\begin{array}{l}11,167(98.63 \%) \\
155(1.37 \%)\end{array}$ & $\begin{array}{l}89,817(98.91 \%) \\
993(1.09 \%)\end{array}$ & 0.0247 \\
\hline $\begin{array}{l}\text { Previous PCI/ } \\
\text { PTCA }\end{array}$ & 0 & $\begin{array}{l}\text { No } \\
\text { Yes }\end{array}$ & $\begin{array}{l}120,759(97.87 \%) \\
2630(2.13 \%)\end{array}$ & $\begin{array}{l}20,854(98.10 \%) \\
403(1.90 \%)\end{array}$ & $\begin{array}{l}11,044(97.54 \%) \\
278(2.46 \%)\end{array}$ & $\begin{array}{l}88,861(97.85 \%) \\
1949(2.15 \%)\end{array}$ & 0.0033 \\
\hline $\begin{array}{l}\text { Pre-Op renal } \\
\text { insufficiency }\end{array}$ & 0 & $\begin{array}{l}\text { No } \\
\text { Yes }\end{array}$ & $\begin{array}{l}122,571(99.34 \%) \\
818(0.66 \%)\end{array}$ & $\begin{array}{l}21,092(99.22 \%) \\
165(0.78 \%)\end{array}$ & $\begin{array}{l}11,204(98.96 \%) \\
118(1.04 \%)\end{array}$ & $\begin{array}{l}90,275(99.41 \%) \\
535(0.59 \%)\end{array}$ & $<0.0001$ \\
\hline $\begin{array}{l}\text { Pre-Op obstruc- } \\
\text { tive sleep apnea }\end{array}$ & 0 & $\begin{array}{l}\text { No } \\
\text { Yes }\end{array}$ & $\begin{array}{l}76,839(62.27 \%) \\
46,550(37.73 \%)\end{array}$ & $\begin{array}{l}12,780(60.12 \%) \\
8477(39.88 \%)\end{array}$ & $\begin{array}{l}6695(59.13 \%) \\
4627(40.87 \%)\end{array}$ & $\begin{array}{l}57,364(63.17 \%) \\
33,446(36.83 \%)\end{array}$ & $<0.0001$ \\
\hline $\begin{array}{l}\text { Current smoker } \\
\text { within } 1 \text { year }\end{array}$ & 0 & $\begin{array}{l}\text { No } \\
\text { Yes }\end{array}$ & $\begin{array}{l}112,261(90.98 \%) \\
11,128(9.02 \%)\end{array}$ & $\begin{array}{l}19,455(91.52 \%) \\
1802(8.48 \%)\end{array}$ & $\begin{array}{l}10,352(91.43 \%) \\
970(8.57 \%)\end{array}$ & $\begin{array}{l}82,454(90.80 \%) \\
8356(9.20 \%)\end{array}$ & 0.0009 \\
\hline $\begin{array}{l}\text { Pre-Op therapeu- } \\
\text { tic anticoagula- } \\
\text { tion }\end{array}$ & 0 & $\begin{array}{l}\text { No } \\
\text { Yes }\end{array}$ & $\begin{array}{l}120,671(97.80 \%) \\
2718(2.20 \%)\end{array}$ & $\begin{array}{l}20,762(97.67 \%) \\
495(2.33 \%)\end{array}$ & $\begin{array}{l}10,993(97.09 \%) \\
329(2.91 \%)\end{array}$ & $\begin{array}{l}88,916(97.91 \%) \\
1894(2.09 \%)\end{array}$ & $<0.0001$ \\
\hline $\begin{array}{l}\text { Pre-Op venous } \\
\text { stasis }\end{array}$ & 0 & $\begin{array}{l}\text { No } \\
\text { Yes }\end{array}$ & $\begin{array}{l}122,025(98.89 \%) \\
1364(1.11 \%)\end{array}$ & $\begin{array}{l}21,072(99.13 \%) \\
185(0.87 \%)\end{array}$ & $\begin{array}{l}11,140(98.39 \%) \\
182(1.61 \%)\end{array}$ & $\begin{array}{l}89,813(98.90 \%) \\
997(1.10 \%)\end{array}$ & $<0.0001$ \\
\hline
\end{tabular}

* $p$ values were calculated from $\chi^{2}$ test for categorical variables, and Kruskal-Wallis test for continuous variables

\section{Procedure duration}

The mean procedural duration of laparoscopic RYGB was $107 \pm 60 \mathrm{~min}: 126 \pm 61,137 \pm 59$, and $97 \pm 55 \mathrm{~min}$ for resident, fellow, and non-trainee participants, respectively ( $p$ value $<0.0001)$. After adjusting for differences in baseline characteristics (including age, gender, BMI), fellow participation was independently associated with increased procedural duration (Ratio in duration 1.39, 95\% CI 1.37-1.41 over non-trainee participants, and Ratio 1.07, 95\% CI 1.05-1.09 over resident participants). For robotic RYGB, the mean operative duration was $136 \pm 64 \mathrm{~min}: 138 \pm 70$, $178 \pm 72$, and $132 \pm 59 \mathrm{~min}$ for resident, fellow, and nontrainee participants, respectively ( $p$ value $<0.0001$ ). After adjusting for differences in baseline characteristics, fellow participation was independently associated with increased procedural duration (Ratio in duration 1.28 , 95\% CI 1.23-1.38 over non-trainee participants, and Ratio 1.17 , 95\% CI 1.10-1.24 over resident participants). For laparoscopic SG, the mean operative duration was $66 \pm 4 \mathrm{~min}$ : $80 \pm 4,81 \pm 42$, and $62 \pm 36$ min for resident, fellow, and non-trainee participation, respectively ( $p$ value $<0.0001$ ) After adjusting for differences in baseline characteristics, fellow participation was independently associated with increased procedural duration (Ratio in duration 1.30, 95\% CI 1.29-1.32 over non-trainee participants, and Ratio 1.02,
95\% CI 1.01-1.03 over resident participants). For robotic SG, the mean operative duration was $93 \pm 51$ min: $97 \pm 49$, $100 \pm 52$, and $91 \pm 51 \mathrm{~min}$ for resident, fellow, and nontrainee participation, respectively ( $p$ value $<0.0001$ ). After adjusting for differences in baseline characteristics, fellow participation was independently associated with increased procedural duration when compared to non-trainee participants (Ratio in duration 1.08, 95\% CI 1.04-1.1) but not when compared to resident participants (Ratio 1.03, 95\% CI 0.98-1.08).

\section{Impact of fellow involvement on RYGB}

Fellows participated in 4188 total laparoscopic RYGB procedures. In cases with fellow participation, the mortality, 30-day re-operation, and 30-day readmission rates were $0.12 \%, 2.17 \%, 6.4 \%$, respectively $(p=0.59,0.60,0.16$, respectively, for all unadjusted comparisons with resident trainee and non-trainee groups) (Table 2). 30-day complication rate was highest in fellow cases (5.68\% compared to $4.75 \%$ for residents and $4.32 \%$ for the non-trainee group, $p=0.0003$ ). For individually examined postoperative complications, higher rate was seen only for SSI and UTI (Table 2). After adjusting for baseline characteristics, fellow participation was associated with higher risk of having a complication but no significant difference in 30-day 
Table 2 30-Day complications after laparoscopic RYGB based on assistant participation

\begin{tabular}{|c|c|c|c|c|c|c|c|}
\hline Variables & Missing & Levels & Total & Resident $(\mathrm{N}=6234)$ & $\begin{array}{l}\text { Minimally inva- } \\
\text { sive surgery fellow } \\
(\mathrm{N}=4188)\end{array}$ & $\begin{array}{l}\text { Non-trainee } \\
(\mathrm{N}=24,932)\end{array}$ & p-values \\
\hline \multirow[t]{2}{*}{ Acute renal failure } & \multirow[t]{2}{*}{0} & No & $35,297(99.84 \%)$ & $6225(99.86 \%)$ & $4183(99.88 \%)$ & $24,889(99.83 \%)$ & 0.6834 \\
\hline & & Yes & $57(0.16 \%)$ & $9(0.14 \%)$ & $5(0.12 \%)$ & $43(0.17 \%)$ & \\
\hline \multirow{2}{*}{$\begin{array}{l}\text { Intra-Op or Post-Op } \\
\text { cardiac arrest }\end{array}$} & \multirow[t]{2}{*}{0} & No & $35,330(99.93 \%)$ & $6229(99.92 \%)$ & $4187(99.98 \%)$ & $24,914(99.93 \%)$ & 0.549 \\
\hline & & Yes & $24(0.07 \%)$ & $5(0.08 \%)$ & $1(0.02 \%)$ & $18(0.07 \%)$ & \\
\hline \multirow[t]{2}{*}{ Stroke } & \multirow[t]{2}{*}{0} & No & $35,351(99.99 \%)$ & $6234(100.00 \%)$ & $4188(100.00 \%)$ & $24,929(99.99 \%)$ & 0.7369 \\
\hline & & Yes & $3(0.01 \%)$ & $0(0.00 \%)$ & $0(0.00 \%)$ & $3(0.01 \%)$ & \\
\hline \multirow[t]{2}{*}{ Peripheral nerve injury } & \multirow[t]{2}{*}{0} & No & $35,350(99.99 \%)$ & $6233(99.98 \%)$ & $4187(99.98 \%)$ & $24,930(99.99 \%)$ & 0.7485 \\
\hline & & Yes & $4(0.01 \%)$ & $1(0.02 \%)$ & $1(0.02 \%)$ & $2(0.01 \%)$ & \\
\hline \multirow{2}{*}{$\begin{array}{l}\text { Progressive renal insuf- } \\
\text { ficiency }\end{array}$} & \multirow[t]{2}{*}{0} & No & $35,308(99.87 \%)$ & $6222(99.81 \%)$ & $4181(99.83 \%)$ & $24,905(99.89 \%)$ & 0.1997 \\
\hline & & Yes & $46(0.13 \%)$ & $12(0.19 \%)$ & $7(0.17 \%)$ & $27(0.11 \%)$ & \\
\hline \multirow[t]{2}{*}{ Pulmonary embolism } & \multirow[t]{2}{*}{0} & No & $35,305(99.86 \%)$ & $6225(99.86 \%)$ & $4181(99.83 \%)$ & $24,899(99.87 \%)$ & 0.8472 \\
\hline & & Yes & $49(0.14 \%)$ & $9(0.14 \%)$ & $7(0.17 \%)$ & $33(0.13 \%)$ & \\
\hline \multirow{2}{*}{$\begin{array}{l}\text { Transfusion Intra-Op/ } \\
\text { Post-Op ( } 72 \mathrm{~h} \text { of } \\
\text { surgery start time) }\end{array}$} & \multirow[t]{2}{*}{0} & No & $34,939(98.83 \%)$ & $6166(98.91 \%)$ & $4142(98.90 \%)$ & $24,631(98.79 \%)$ & 0.6648 \\
\hline & & Yes & $415(1.17 \%)$ & $68(1.09 \%)$ & $46(1.10 \%)$ & $301(1.21 \%)$ & \\
\hline \multirow{2}{*}{$\begin{array}{l}\text { Unplanned admission } \\
\text { to ICU within } 30 \text { days }\end{array}$} & \multirow[t]{2}{*}{0} & No & $34,900(98.72 \%)$ & $6144(98.56 \%)$ & $4122(98.42 \%)$ & $24,634(98.80 \%)$ & 0.0602 \\
\hline & & Yes & $454(1.28 \%)$ & $90(1.44 \%)$ & $66(1.58 \%)$ & $298(1.20 \%)$ & \\
\hline \multirow[t]{2}{*}{ Unplanned intubation } & \multirow[t]{2}{*}{0} & No & $35,247(99.70 \%)$ & $6216(99.71 \%)$ & $4176(99.71 \%)$ & $24,855(99.69 \%)$ & 0.9476 \\
\hline & & Yes & $107(0.30 \%)$ & $18(0.29 \%)$ & $12(0.29 \%)$ & $77(0.31 \%)$ & \\
\hline \multirow{2}{*}{$\begin{array}{l}\text { Post-Op vein thrombo- } \\
\text { sis requiring therapy }\end{array}$} & \multirow[t]{2}{*}{0} & No & $35,293(99.83 \%)$ & $6224(99.84 \%)$ & $4178(99.76 \%)$ & $24,891(99.84 \%)$ & 0.5448 \\
\hline & & Yes & $61(0.17 \%)$ & $10(0.16 \%)$ & $10(0.24 \%)$ & $41(0.16 \%)$ & \\
\hline \multirow[t]{2}{*}{ Wound disruption } & \multirow[t]{2}{*}{0} & No & $35,330(99.93 \%)$ & $6229(99.92 \%)$ & $4187(99.98 \%)$ & $24,914(99.93 \%)$ & 0.5519 \\
\hline & & Yes & $24(0.07 \%)$ & $5(0.08 \%)$ & $1(0.02 \%)$ & $18(0.07 \%)$ & \\
\hline \multirow{2}{*}{$\begin{array}{l}\text { Post-Op deep incisional } \\
\text { SSI occurrences }\end{array}$} & \multirow[t]{2}{*}{0} & No & $35,304(99.86 \%)$ & $6227(99.89 \%)$ & $4185(99.93 \%)$ & $24,892(99.84 \%)$ & 0.2955 \\
\hline & & Yes & $50(0.14 \%)$ & $7(0.11 \%)$ & $3(0.07 \%)$ & $40(0.16 \%)$ & \\
\hline \multirow{2}{*}{$\begin{array}{l}\text { Post-Op organ/space } \\
\text { SSI occurrences }\end{array}$} & 0 & No & $35,224(99.63 \%)$ & $6213(99.66 \%)$ & $4169(99.55 \%)$ & $24,842(99.64 \%)$ & 0.5952 \\
\hline & & Yes & $130(0.37 \%)$ & $21(0.34 \%)$ & $19(0.45 \%)$ & $90(0.36 \%)$ & \\
\hline Post-Op pneumonia & 0 & No & $35,216(99.61 \%)$ & $6211(99.63 \%)$ & $4166(99.47 \%)$ & $24,839(99.63 \%)$ & 0.3282 \\
\hline occurrences & & Yes & $138(0.39 \%)$ & $23(0.37 \%)$ & $22(0.53 \%)$ & $93(0.37 \%)$ & \\
\hline Post-Op septic shock & 0 & No & $35,308(99.87 \%)$ & $6223(99.82 \%)$ & $4183(99.88 \%)$ & $24,902(99.88 \%)$ & 0.535 \\
\hline occurrences & & Yes & $46(0.13 \%)$ & $11(0.18 \%)$ & $5(0.12 \%)$ & $30(0.12 \%)$ & \\
\hline Post-Op superficial & 0 & No & $35,015(99.04 \%)$ & $6171(98.99 \%)$ & $4126(98.52 \%)$ & $24,718(99.14 \%)$ & 0.0006 \\
\hline $\begin{array}{l}\text { incisional SSI occur- } \\
\text { rences }\end{array}$ & & Yes & $339(0.96 \%)$ & $63(1.01 \%)$ & $62(1.48 \%)^{\mathrm{NT}, \mathrm{R}}$ & $214(0.86 \%)$ & \\
\hline Post-Op urinary tract & 0 & No & $35,195(99.55 \%)$ & $6188(99.26 \%)$ & $4155(99.21 \%)$ & $24,852(99.68 \%)$ & $<0.0001$ \\
\hline infection occurrences & & Yes & $159(0.45 \%)$ & $46(0.74 \%)^{\mathrm{NT}}$ & $33(0.79 \%)^{\mathrm{NT}}$ & $80(0.32 \%)$ & \\
\hline Ventilator $>48 \mathrm{~h}$ occur- & 0 & No & $35,285(99.80 \%)$ & $6217(99.73 \%)$ & $4184(99.90 \%)$ & $24,884(99.81 \%)$ & 0.1262 \\
\hline rences & & Yes & $69(0.20 \%)$ & $17(0.27 \%)$ & $4(0.10 \%)$ & $48(0.19 \%)$ & \\
\hline Anastomotic/staple line & 0 & No & $35,150(99.42 \%)$ & $6206(99.55 \%)$ & $4164(99.43 \%)$ & $24,780(99.39 \%)$ & 0.3261 \\
\hline & & Yes & $204(0.58 \%)$ & $28(0.45 \%)$ & $24(0.57 \%)$ & $152(0.61 \%)$ & \\
\hline Any complication & 0 & No & $33,743(95.44 \%)$ & $5938(95.25 \%)$ & $3950(94.32 \%)$ & $23,855(95.68 \%)$ & 0.0003 \\
\hline & & Yes & $1611(4.56 \%)$ & $296(4.75 \%)$ & $238(5.68 \%)^{\mathrm{NT}}$ & $1077(4.32 \%)$ & \\
\hline
\end{tabular}

ICU intensive care unit, SSI surgical site infection

${ }^{\mathrm{NT}}$ Statistical significance for unadjusted $p$ values calculated from $\chi^{2}$ test comparing to non-trainee

${ }^{\mathrm{R}}$ Statistical significance for unadjusted $p$ values calculated from $\chi^{2}$ test comparing to resident 
re-operation, anastomotic/staple line leak, or readmission when compared to the resident or non-trainee groups (Table 4). After further adjusting for operative duration, the negative impact of fellow participation on having any complication was no longer observed (OR 1.15, 95\% CI 0.99-1.34 over non-trainee, and OR 1.14, 95\% CI 0.96-1.34 over resident participants) (Table 5). A surgical fellow participated in a total of 268 robotic RYGB cases. With fellow participation, there was no increased rate of mortality $(0 \%)$, re-operation $(1.87 \%)$, and readmission $(7.46 \%)(p=1,0.69$, 0.35 , respectively, for all unadjusted comparisons with resident trainees and non-trainee participants). The 30-day complication rate was highest in fellow cases (5.22 vs. $2.36 \%$ for residents and $2.56 \%$ for non-trainee, $p=0.04$ ). From all the individual complications analyzed, only UTI was higher in the fellow group ( 1.12 vs. $0.34 \%$ for residents and $0.09 \%$ for non-trainee, $p=0.01$ ). After adjusting for baseline characteristics, fellow involvement was independently associated with the development of postoperative complications compared to non-trainees (Table 4). With the inclusion of operative duration in the adjustment modeling, the negative impact of fellow participation on postoperative complications was no longer observed (OR 1.70, 95\% CI 0.92-3.15 over nontrainee group, and OR 2.37, 95\% CI 0.93-6.25 over resident group) (Table 5).

\section{Impact of fellow involvement on sleeve gastrectomy procedures}

Fellows participated in 6331 laparoscopic SG procedures. With fellow participation, the mortality $(0.09 \%)$ and re-operation rates $(0.88 \%)$ were not significantly different $(p=0.87$, 0.88 , respectively). However, there was a significant increase in the 30-day re-intervention ( 1.17 vs. $1.40 \%$ for residents and $0.98 \%$ for non-trainee, $p<0.0001$ ) and readmission rates (3.85 vs. $3.82 \%$ for residents and $3.15 \%$ for non-trainee, $p<0.0001)$. The 30 -day complication rates were highest in fellow group (2.61 vs. $2.53 \%$ for residents and $2.00 \%$ for non-trainee, $p<0.0001)$. Specifically, when comparing fellow to non-trainee, there was an increased incidence in progressive renal insufficiency, pulmonary emboli, postoperative superficial SSI, and UTI (Table 3). After adjusting for baseline characteristics, fellow participation was independently associated with a higher risk of 30-day readmission and complications when compared to a non-trainee (Table 4). However, after further adjusting for operative duration, the negative impact of fellow participation on having any complication or readmission was no longer observed (Table 5). Fellows participated in 535 robotic SG surgeries. With fellow participation there was no significant impact on mortality, 30-day re-operation, and 30-day readmission (rates were $0 \%, 0.93 \%, 5.23 \%$, and $p=0.51,0.59,0.23$, respectively, for all unadjusted comparisons with resident trainee and non-trainee groups). Furthermore, 30-day events were similar in fellow procedures (Table 4).

\section{Impact of resident participation on RYGB procedures}

A total of 6234 laparoscopic RYGB were performed with a resident participant. After adjusting for baseline characteristics, the resident group did not have an increased rate of 30-day readmissions, 30-day re-operations, developing any complication or anastomotic/staple line leaks (Table 4). Operative duration for resident cases was longer when compared to the non-trainee group, however, it was shorter than for the fellow group (Ratio in duration 1.30, 95\% CI 1.28-1.31 compared to non-trainee group, and Ratio in duration 0.93 , 95\% CI $0.92-0.95$ compared to fellow group). A total of 297 robotic RYGB were performed with a resident. After adjusting for baseline characteristics, the resident group did not have an increased risk of readmissions, reoperations, developing any complication or anastomotic/ staple line leak when compared to the non-trainee or fellow groups (Table 4).

\section{Impact of resident participation on sleeve gastrectomy procedures}

A total of 14,100 laparoscopic SG procedures were performed with a resident participant. After adjusting for baseline characteristics, the resident group had a higher risk of readmissions, anastomotic/staple line leak, and complications than the non-trainee group (Table 4). The negative impact of resident participation on increased complication risk, anastomotic/staple line leak, readmission, and re-intervention remained even after adjusting for operative duration (Table 5). Residents participated in 626 of robotic SG procedures. After adjusting for baseline characteristics, the resident group did not have statistically significant impact on the risk of any complication within 30 days, anastomotic/ staple line leak, 30-day readmission, 30-day re-operation, or 30-day re-intervention (Table 4).

\section{Discussion}

With the increasing number of bariatric procedures performed as well as increase in the number of available bariatric fellowships, it has become increasingly important to balance patient safety as well as the training of the next generation of bariatric surgeons [25]. Furthermore, with the advancement of robotic bariatric surgery, and the changing bariatric procedural landscape, the paradigm of the modern surgeon training is further complicated [26]. There is limited literature on the impact of trainee participation during 
Table 3 30-Day complications after laparoscopic SG based on assistant participation

\begin{tabular}{|c|c|c|c|c|c|c|c|}
\hline Variables & Missing & Levels & Total & Resident $(N=14,100)$ & $\begin{array}{l}\text { Minimally inva- } \\
\text { sive surgery fellow } \\
(N=6331)\end{array}$ & $\begin{array}{l}\text { Non-trainee } \\
(N=59,286)\end{array}$ & $p$ values \\
\hline \multirow[t]{2}{*}{ Acute renial failure } & \multirow[t]{2}{*}{0} & No & $79662(99.93 \%)$ & $14090(99.93 \%)$ & $6327(99.94 \%)$ & $59245(99.93 \%)$ & 0.6834 \\
\hline & & Yes & $55(0.07 \%)$ & $10(0.07 \%)$ & $4(0.06 \%)$ & $41(0.07 \%)$ & \\
\hline \multirow{2}{*}{$\begin{array}{l}\text { Intra-Op or Post-Op } \\
\text { cardiac arrest }\end{array}$} & \multirow[t]{2}{*}{0} & No & $79,685(99.96 \%)$ & $14,092(99.94 \%)$ & $6328(99.95 \%)$ & $59,265(99.96 \%)$ & 0.4866 \\
\hline & & Yes & $32(0.04 \%)$ & $8(0.06 \%)$ & $3(0.05 \%)$ & $21(0.04 \%)$ & \\
\hline \multirow[t]{2}{*}{ Stroke } & \multirow[t]{2}{*}{0} & No & 79,708 (99.99\%) & $14,099(99.99 \%)$ & $6328(99.95 \%)$ & $59,281(99.99 \%)$ & 0.045 \\
\hline & & Yes & $9(0.01 \%)$ & $1(0.01 \%)$ & $3(0.05 \%)^{\mathrm{NT}}$ & $5(0.01 \%)$ & \\
\hline \multirow{2}{*}{$\begin{array}{l}\text { Peripheral nerve } \\
\text { injury }\end{array}$} & \multirow[t]{2}{*}{0} & No & $79,717(100.00 \%)$ & $14,100(100.00 \%)$ & $6331(100.00 \%)$ & $59,286(100.00 \%)$ & 1 \\
\hline & & Yes & $0(0.00 \%)$ & $0(0.00 \%)$ & $0(0.00 \%)$ & $0(0.00 \%)$ & \\
\hline \multirow{2}{*}{$\begin{array}{l}\text { Progressive renal } \\
\text { insufficiency }\end{array}$} & \multirow[t]{2}{*}{0} & No & $79,653(99.92 \%)$ & $14,082(99.87 \%)$ & $6322(99.86 \%)$ & $59,249(99.94 \%)$ & 0.0094 \\
\hline & & Yes & $64(0.08 \%)$ & $18(0.13 \%)^{\mathrm{NT}}$ & $9(0.14 \%)^{\mathrm{NT}}$ & $37(0.06 \%)$ & \\
\hline \multirow{2}{*}{ Pulmonary embolism } & \multirow[t]{2}{*}{0} & No & $79,642(99.91 \%)$ & $14,082(99.87 \%)$ & $6319(99.81 \%)$ & $59,241(99.92 \%)$ & 0.007 \\
\hline & & Yes & $75(0.09 \%)$ & $18(0.13 \%)$ & $12(0.19 \%)^{\mathrm{NT}}$ & $45(0.08 \%)$ & \\
\hline \multirow{2}{*}{$\begin{array}{l}\text { Transfusion Intra-op/ } \\
\text { Post-op ( } 72 \mathrm{~h} \text { of } \\
\text { surgery start time) }\end{array}$} & \multirow[t]{2}{*}{0} & No & $79,268(99.44 \%)$ & $14,010(99.36 \%)$ & $6290(99.35 \%)$ & $58,968(99.46 \%)$ & 0.2246 \\
\hline & & Yes & $449(0.56 \%)$ & $90(0.64 \%)$ & $41(0.65 \%)$ & $318(0.54 \%)$ & \\
\hline \multirow{2}{*}{$\begin{array}{l}\text { Unplanned admis- } \\
\text { sion to ICU within } \\
30 \text { days }\end{array}$} & \multirow[t]{2}{*}{0} & No & $79,273(99.44 \%)$ & $14,016(99.40 \%)$ & $6298(99.48 \%)$ & $58,959(99.45 \%)$ & 0.7558 \\
\hline & & Yes & $444(0.56 \%)$ & $84(0.60 \%)$ & $33(0.52 \%)$ & $327(0.55 \%)$ & \\
\hline \multirow[t]{2}{*}{ Unplanned intubation } & \multirow[t]{2}{*}{0} & No & $79,620(99.88 \%)$ & $14,080(99.86 \%)$ & $6325(99.91 \%)$ & $59,215(99.88 \%)$ & 0.6483 \\
\hline & & Yes & $97(0.12 \%)$ & $20(0.14 \%)$ & $6(0.09 \%)$ & $71(0.12 \%)$ & \\
\hline \multirow{2}{*}{$\begin{array}{l}\text { Post-Op vein throm- } \\
\text { bosis requiring } \\
\text { therapy }\end{array}$} & \multirow[t]{2}{*}{0} & No & $79,576(99.82 \%)$ & $14,070(99.79 \%)$ & $6316(99.76 \%)$ & $59,190(99.84 \%)$ & 0.2152 \\
\hline & & Yes & $141(0.18 \%)$ & $30(0.21 \%)$ & $15(0.24 \%)$ & $96(0.16 \%)$ & \\
\hline \multirow[t]{2}{*}{ Wound disruption } & \multirow[t]{2}{*}{0} & No & $79,700(99.98 \%)$ & $14,098(99.99 \%)$ & $6331(100.00 \%)$ & $59,271(99.97 \%)$ & 0.3632 \\
\hline & & Yes & $17(0.02 \%)$ & $2(0.01 \%)$ & $0(0.00 \%)$ & $15(0.03 \%)$ & \\
\hline \multirow{2}{*}{$\begin{array}{l}\text { Post-Op deep inci- } \\
\text { sional SSI occur- } \\
\text { rences }\end{array}$} & \multirow[t]{2}{*}{0} & No & $79,701(99.98 \%)$ & $14,099(99.99 \%)$ & $6329(99.97 \%)$ & $59,273(99.98 \%)$ & 0.4703 \\
\hline & & Yes & $16(0.02 \%)$ & $1(0.01 \%)$ & $2(0.03 \%)$ & $13(0.02 \%)$ & \\
\hline \multirow{2}{*}{$\begin{array}{l}\text { Post-Op organ/space } \\
\text { SSI occurrences }\end{array}$} & 0 & No & $79,581(99.83 \%)$ & $14,068(99.77 \%)$ & $6320(99.83 \%)$ & $59,193(99.84 \%)$ & 0.1931 \\
\hline & & Yes & $136(0.17 \%)$ & $32(0.23 \%)$ & $11(0.17 \%)$ & $93(0.16 \%)$ & \\
\hline Post-Op pneumonia & 0 & No & $79,618(99.88 \%)$ & $14,081(99.87 \%)$ & $6326(99.92 \%)$ & $59,211(99.87 \%)$ & 0.5499 \\
\hline occurrences & & Yes & $99(0.12 \%)$ & $19(0.13 \%)$ & $5(0.08 \%)$ & $75(0.13 \%)$ & \\
\hline Post-Op septic shock & 0 & No & $79,688(99.96 \%)$ & $14,092(99.94 \%)$ & $6329(99.97 \%)$ & $59,267(99.97 \%)$ & 0.3962 \\
\hline occu & & Yes & $29(0.04 \%)$ & $8(0.06 \%)$ & $2(0.03 \%)$ & $19(0.03 \%)$ & \\
\hline Post-Op superficial & 0 & No & $79,528(99.76 \%)$ & $14,058(99.70 \%)$ & $6309(99.65 \%)$ & $59,161(99.79 \%)$ & 0.0274 \\
\hline $\begin{array}{l}\text { incisional SSI occur- } \\
\text { rences }\end{array}$ & & Yes & $189(0.24 \%)$ & $42(0.30 \%)$ & $22(0.35 \%)^{\mathrm{NT}}$ & $125(0.21 \%)$ & \\
\hline & 0 & No & $79,494(99.72 \%)$ & $14,046(99.62 \%)$ & $6301(99.53 \%)$ & $59,147(99.77 \%)$ & 0.0001 \\
\hline $\begin{array}{l}\text { infection occur- } \\
\text { rences }\end{array}$ & & Yes & $223(0.28 \%)$ & $54(0.38 \%)^{\mathrm{NT}}$ & $30(0.47 \%)^{\mathrm{NT}}$ & $139(0.23 \%)$ & \\
\hline Ventilator $>48 \mathrm{~h}$ & 0 & No & $79,671(99.94 \%)$ & $14,089(99.92 \%)$ & $6328(99.95 \%)$ & $59,254(99.95 \%)$ & 0.5194 \\
\hline occurrences & & Yes & $46(0.06 \%)$ & $11(0.08 \%)$ & $3(0.05 \%)$ & $32(0.05 \%)$ & \\
\hline Anastomotic/staple & 0 & No & $79,454(99.67 \%)$ & $14,035(99.54 \%)$ & $6310(99.67 \%)$ & $59,109(99.70 \%)$ & 0.0104 \\
\hline line leak & & Yes & $263(0.33 \%)$ & $65(0.46 \%)^{\mathrm{NT}}$ & $21(0.33 \%)$ & $177(0.30 \%)$ & \\
\hline Any complication & 0 & No & $78,012(97.86 \%)$ & $13,743(97.47 \%)$ & $6166(97.39 \%)$ & $58,103(98.00 \%)$ & $<0.0001$ \\
\hline & & Yes & $1705(2.14 \%)$ & $357(2.53 \%)^{\mathrm{NT}}$ & $165(2.61 \%)^{\mathrm{NT}}$ & $1183(2.00 \%)$ & \\
\hline
\end{tabular}

ICU intensive care unit, SSI surgical site infection

${ }^{\mathrm{NT}}$ Statistical significance for unadjusted $p$ values calculated from $\chi^{2}$ test comparing to non-trainee

${ }^{\mathrm{R}}$ Statistical significance for unadjusted $p$ values calculated from $\chi^{2}$ test comparing to resident 


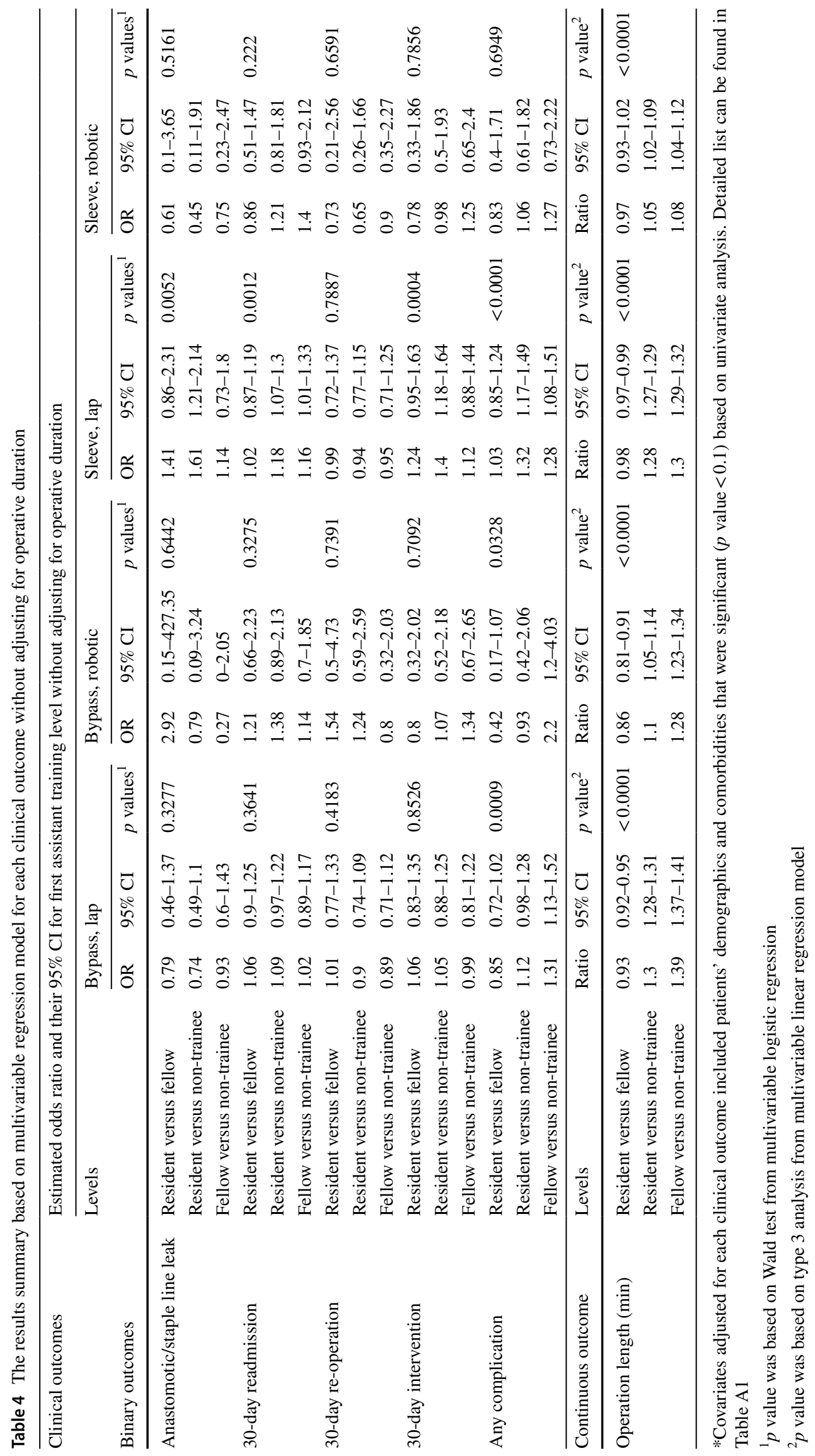




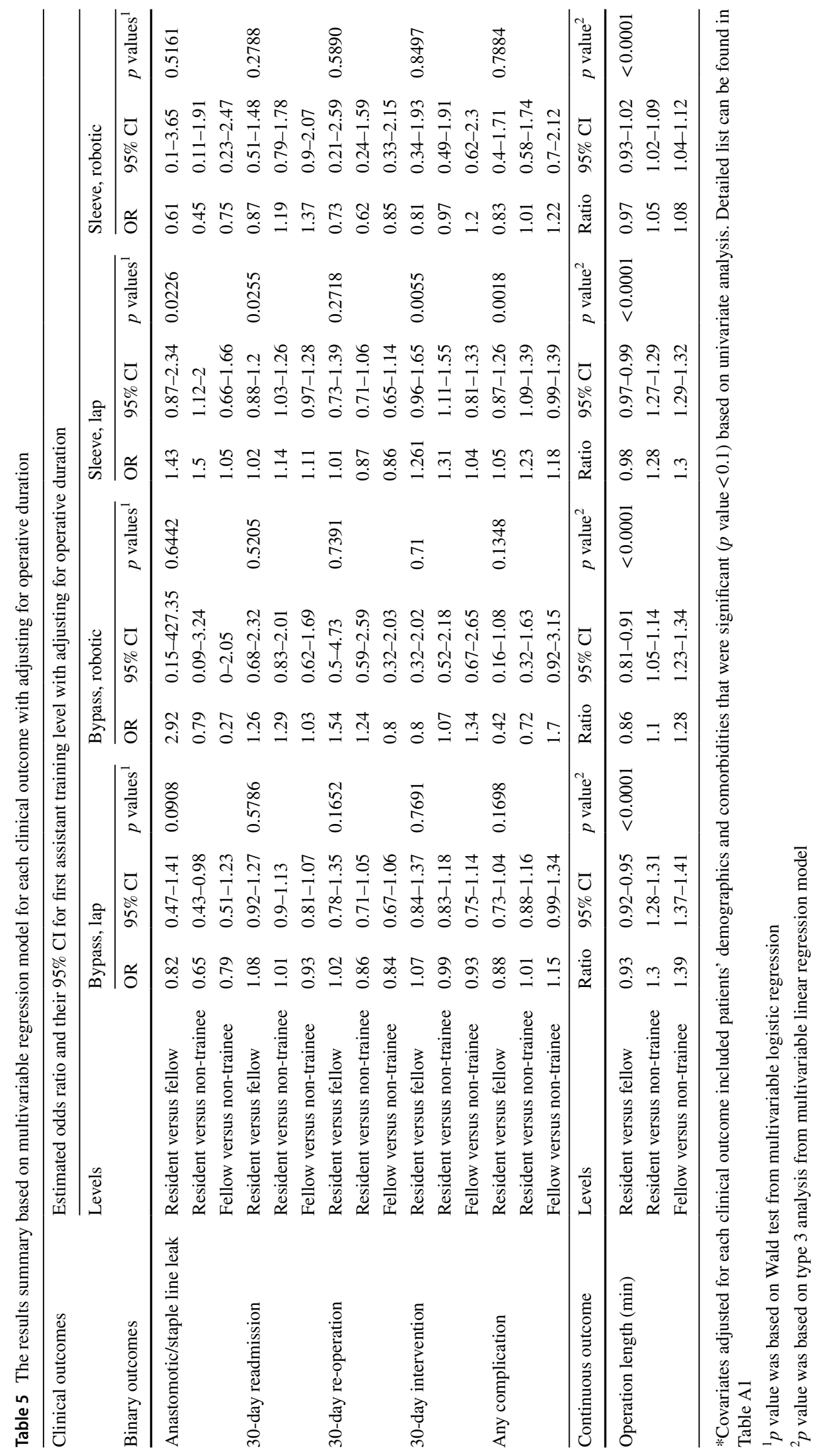


bariatric surgery, particularly as it pertains to differences between trainee levels, and how this impact can vary for different procedure types and approaches.

Our study showed that there was a significant increase in duration of surgery with fellow participation for laparoscopic and robotic bariatric procedures when compared to the non-trainee group. Furthermore, fellow participation was associated with longer operative duration when compared to resident participation for most procedure types examined. This is consistent with previous studies in terms of the impact of trainees on operative duration [18, 27]. In contrast to our results, others have suggested that while trainee participation is associated with worse clinical outcomes and longer operative duration, there is no significant difference between residents and fellows [17].

Laparoscopic RYGB is a complex procedure that requires advanced skills and understandings of key surgical steps [28]. In our study, fellow participation during laparoscopic RYGB was associated with an increased risk of developing complications when compared to non-trainees. Interestingly, resident participation was not associated with an increased complication risk for laparoscopic RYGB in relation to nontrainee or fellow participants. The safety of resident participation in laparoscopic RYGB procedures has been previously noted by others. Iordens et al. compared postoperative complications, return to emergency room, and readmission rates following laparoscopic RYGB performed with a resident or a second attending surgeon and noted no significant difference between the two groups [29]. Similarly, another study conducted by Fanous et al. further suggested that there was no difference in intraoperative complications, length of stay, 30-day adverse outcomes, or 1-year rate of re-operation between a physician assistant and a senior resident [30]. Based on previous literature as well as our findings, it appears that resident participation is overall safe for the bariatric patient undergoing laparoscopic RYGB. It is important to note that the lower operative duration for residents compared to fellows suggests - as it would be expected-that the degree of intraoperative participation may be different between the two groups.

Laparoscopic SG procedures have been gaining popularity at a rapid rate [3]. We identified an increased complication rate when laparoscopic SG was performed with a fellow or resident. This finding is different compared to what others have reported. Aminian et al., using nationwide data from 2010 to 2012, found no significant increase in complication rate with fellow participation for laparoscopic SG [18]. This difference in findings could be possibly explained by the increase in popularity of SG over time. Importantly, our study suggests that fellow participation was not associated with a higher readmission, re-intervention, or anastomotic/ staple line leak rate. On the contrary, resident participation was independently associated with a higher rate of serious complications, including anastomotic/staple line leaks. While previous studies have suggested increased morbidity with resident involvement in SG procedures, this impact has been limited to cardiac events and UTI [17]. The mechanism of such increase in anastomotic leaks and other complications of laparoscopic SG with resident involvement remains unclear. It is plausible that given the seemingly less complex technical nature of this procedure, resident participation may be in greater degree compared to laparoscopic RYGB, allowing for the limitations in the understanding of the technique or skillset to translate into measurable clinical outcomes. This is further supported by the similarity in operative duration between fellow and resident participants during SG procedures.

Robotic RYGB surgery is a newly emerging option for bariatric patients. In our study, robotic RYGB performed with a fellow was associated with the development of complications, primarily UTI, when compared with non-trainee participation. Both for laparoscopic SG and RYGB, after accounting for operative duration, the negative impact of fellow participation was no longer observed. This likely indicates that with technical proficiency and experience, the fellow impact is minimized. Furthermore, robotic surgery performed with a resident appeared to be safe for the patient and may be a promising platform for training.

One of the main limitations in our study is that it did not account for the competency level of the primary surgeon. Furthermore, prolonged duration may be an indication of higher procedural complexity. Fellowship training is often found at academic institutions and tertiary care centers where fellows participate in procedures with more complicated patients [18]. We attempted to account for this using comorbidities, gender, and BMI, but detailed surgical history was not available in the database. Another significant limitation is that the resident category is not further clarified in terms of post-graduate year. It is likely that resident skill level contributed to the outcome of the surgery as well as the amount of intraoperative participation. Importantly, the degree of participation of the trainee or non-trainee assistant is unknown and could bias our results.

Future research will be geared toward further investigating the extent of trainee involvement during the various bariatric procedures. One area of investigation is whether fellow operative speed and impact on outcomes varies between the initial portion of their training and the latter portion. Furthermore, categorizing the resident training level may help elucidate whether the negative association with resident participation in laparoscopic SG is merely a function of lack of exposure and experience with laparoscopic techniques. A metric-either practical, theoretical, or a combination of both-may be developed to stratify the ability level of the trainee. This pre-determined ability level may help guide the extent of trainee participation during the surgery. While this 
certainly will require several years to establish and validate, future efforts in this arena will be valuable for advancing the field.

\section{Conclusions}

While training the next generation of bariatric surgeons is crucial to ensuring the field continues to progress and flourish, it is important to maintain patient safety. Although minimally invasive/bariatric fellow training is associated with higher overall complication risk, there is no such impact on major safety benchmarks, including anastomotic/staple line leak rate and 30-day re-operations. Importantly, the impact of fellow participation on safety measures appeared to be confounded by the procedural duration, suggesting that with experience and subsequent improvement in procedural duration, the negative impact may be likely reversed. Resident participation in laparoscopic SG appears to have a significant negative impact on patient safety that remained when accounting for procedural duration. Operative involvement of trainees in bariatric surgery leads to different outcomes based on trainee level, and this needs to be considered during the programmatic design of bariatric centers and educational curricula.

Acknowledgements We acknowledge the biostatistical consultation and support provided by the Biostatistical Consulting Core at School of Medicine, Stony Brook University.

Funding No industry or other external funding was used for this research.

\section{Compliance with ethical standards}

Disclosures Dr. Aurora D. Pryor receives Honoraria for speaking for Ethicon, Inc., Medtronic, Stryker, and Gore, and is a Consultant for Medicines Company, Merck, Intuitive, BAROnova and Obalon Therapeutics and has ownership interest in TransEnterix. Dr. Konstantinos Spaniolas is on the Advisory Board for Mallinckrodt and received a Research Grant from Merck. Drs. Iliya Goldberg, Jie Yang, Jihye Park, Salvatore Docimo, Andrew Bates, and Mark A. Talamini have no conflicts of interest or financial ties to disclose.

\section{References}

1. Meek CL, Lewis HB, Reimann F, Gribble FM, Park AJ (2016) The effect of bariatric surgery on gastrointestinal and pancreatic peptide hormones. Peptides 77:28-37

2. Piché M-E, Thorin-Trescases N, Auclair A, Marceau S, Martin J, Fortier A, Thorin E, Poirier P (2017) Bariatric surgery-induced lower angiopoietin-like 2 protein is associated with improved cardiometabolic profile. Can J Cardiol 33(8):1044-1051

3. Spaniolas K, Kasten KR, Brinkley J, Sippey ME, Mozer A, Chapman WH, Pories WJ (2015) The changing bariatric surgery landscape in the USA. Obes Surg 25:1544-1546
4. Hsu GP, Morton JM, Jin L, Safadi BY, Satterwhite TS, Curet MJ (2005) Laparoscopic Roux-en-Y gastric bypass: differences in outcome between attendings and assistants of different training backgrounds. Obes Surg 15:1104-1110

5. Sánchez-Santos R, Masdevall C, Baltasar A, Martínez-Blázquez C, García R, de Gordejuela A, Ponsi E, Sánchez-Pernaute A, Vesperinas G, Del Castillo D, Bombuy E, Durán-Escribano C, Ortega L, Ruiz de Adana JC, Baltar J, Maruri I, GarcíaBlázquez E, Torres A (2009) Short- and mid-term outcomes of sleeve gastrectomy for morbid obesity: the experience of the Spanish National Registry. Obes Surg 19:1203-1210

6. Kim PS, Telem DA, Altieri MS, Talamini M, Yang J, Zhang Q, Pryor AD (2015) Bariatric outcomes are significantly improved in hospitals with fellowship council-accredited bariatric fellowships. J Gastrointest Surg 19(4):594-597

7. Ballantyne GH, Ewing D, Capella RF, Capella JF, Davis D, Schmidt HJ, Wasielewski A, Davies RJ (2005) The learning curve measured by operating times for laparoscopic and open gastric bypass: roles of surgeon's experience, institutional experience, body mass index and fellowship training. Obes Surg 15:172-182

8. Chatterjee A, Pyfer B, Chen L, Czerniecki B, Tchou J, Fisher C (2015) Resident and fellow participation in breast surgery: an American College of Surgeons NSQIP clinical outcomes analysis. J Am Coll Surg 221(5):988-994

9. Advani V, Ahad S, Gonczy C, Markwell S, Hassan I (2012) Does resident involvement effect surgical times and complication rates during laparoscopic appendectomy for uncomplicated appendicitis? An analysis of 16,849 cases from the ACS-NSQIP. Am J Surg 203(3):347-352

10. Iannuzzi JC, Chandra A, Rickles AS, Kumar NG, Kelly KN, Gillespie DL, Monson JR, Fleming FJ (2013) Resident involvement is associated with worse outcomes after major lower extremity amputation. J Vasc Surg 58:827-831

11. Feeney T, Price LL, Chen L, Graham R, Chatterjee A (2017) Resident and fellow participation in thyroid and parathyroid surgery: an ACS-NSQIP clinical outcomes analysis. J Surg Res 220:346-352

12. Jordan SW, Mioton LM, Smetona J, Aggarwal A, Wang E, Dumanian GA, Kim JY (2013) Resident involvement and plastic surgery outcomes: an analysis of 10,356 patients from the American College of Surgeons National Surgical Quality Improvement Program database. Plast Reconstr Surg 131(4):763-773

13. Hernández-Irizarry R, Zendejas B, Ali SM, Lohse CM, Farley DR (2012) Impact of resident participation on laparoscopic inguinal hernia repairs: are residents slowing us down? J Surg Educ 69(6):746-752

14. Raval MV, Wang X, Cohen ME, Ingraham AM, Bentrem DJ, Dimick JB, Flynn T, Hall BL, Ko CY (2011) The influence of resident involvement on surgical outcomes. J Am Coll Surg 212(5):889-898

15. Massenburg BB, Sanati-Mehrizy P, Jablonka EM, Taub PJ (2016) The impact of resident participation in outpatient plastic surgical procedures. Aesthet Plast Surg 40(4):584-591

16. Ejaz A, Spolverato G, Kim Y, Wolfgang CL, Hirose K, Weiss M, Makary MA, Pawlik TM (2015) The impact of resident involvement on surgical outcomes among patients undergoing hepatic and pancreatic resections. Surgery 158(2):323-330

17. Martinovski M, Navratil AL, Zeni T, Jonker M, Ferraro J, Albright J, Cleary RK (2017) Effects of resident or fellow participation in sleeve gastrectomy and gastric bypass: results from the Metabolic and Bariatric Surgery Accreditation and Quality Improvement Program (MBSAQIP). J Am Coll Surg 225(4):S14

18. Aminian A, Chaudhry RM, Khorgami Z, Andalib A, Augustin T, Rodriguez J, Kroh M, Schauer PR, Brethauer SA (2016) A challenge between trainee education and patient safety: does fellow 
participation impact postoperative outcomes following bariatric surgery? Obes Surg 26(9):1999-2005

19. de'Angelis N, Alghamdi S, Renda A, Azoulay D, Brunetti F (2015) Initial experience of robotic versus laparoscopic colectomy for transverse colon cancer: a matched case-control study. World J Surg Oncol 13:295

20. Elli E, Gonzalez-Heredia R, Sarvepalli S, Masrur M (2015) Laparoscopic and robotic sleeve gastrectomy: short- and long-term results. Obes Surg 25:967-974

21. Ecker BL, Maduka R, Ramdon A, Dempsey DT, Dumon KR, Williams NN (2016) Resident education in robotic-assisted vertical sleeve gastrectomy: outcomes and cost-analysis of 411 consecutive cases. Surg Obes Relat Dis 12(2):313-320

22. Vilallonga R, Fort JM, Gonzalez O, Caubet E, Boleko A, Neff KJ, Armengol M (2012) The initial learning curve for robot-assisted sleeve gastrectomy: a surgeon's experience while introducing the robotic technology in a bariatric surgery department. Minim Invasive Surg 2012:347131

23. Starnes CC, Gochnour DC, Hall B, Wilson EB, Snyder BE (2015) The economy of motion of the totally robotic gastric bypass: technique, learning curve, and outcomes of a fellowship-trained, robotic bariatric surgeon. J Laparoendosc Adv Surg Tech A 25(5):411-418

24. Spaniolas K, Laycock WS, Adrales GL, Trus TL (2014) Laparoscopic paraesophageal hernia repair: advanced age is associated with minor but not major morbidity or mortality. J Am Coll Surg 218:1187-1192

25. Kohn GP, Galanko JA, Overby DW, Farrell TM (2010) High case volumes and surgical fellowships are associated with improved outcomes for bariatric surgery patients: a justification of current credentialing initiatives for practice and training. J Am Coll Surg 210:909-918

26. Bindal V, Bhatia P, Dudeja U, Kalhan S, Khetan M, John S, Wadhera $S$ (2015) Review of contemporary role of robotics in bariatric surgery. J Minim Access Surg 11:16-21

27. Krell RW, Birkmeyer NJ, Reames BN, Carlin AM, Birkmeyer JD, Finks JF (2014) Effects of resident involvement on complication rates after laparoscopic gastric bypass. J Am Coll Surg 218(2):253-260

28. Beitner M, Luo Y, Kurian M (2015) Procedural changes to decrease complications in laparoscopic gastric bypass. JSLS 19(1):1-9

29. Iordens GI, Klaassen RA, van Lieshout EM, Cleffken BI, van der Harst E (2012) How to train surgical residents to perform laparoscopic Roux-en-Y gastric bypass safely. World J Surg 36(9):2003-2010

30. Fanous M, Carlin A (2012) Surgical resident participation in laparoscopic Roux-en-Y bypass: is it safe? Surgery 152:21-25 